\title{
Experimental evaluation of two ITS couples of primers for the determination of the fungal diversity
}

Aude STURNY-LECLERE ${ }^{1}$, Cécile GAUTIER ${ }^{1}$, Marion BENAZRA ${ }^{1}$, Marie DESNOS-OLLIVIER ${ }^{1}$, Dea GARCIA-HERMOSO ${ }^{1}$, Françoise DROMER ${ }^{1}$ \& Alexandre ALANIO ${ }^{1,2,3}$

${ }^{1}$ Molecular Mycology Unit, CNRS UMR2000, Institut Pasteur, ${ }^{2}$ Université Sorbonne Paris Cité, ${ }^{3}$ Laboratoire de Parasitologie-Mycologie, Groupe Hospitalier Lariboisière Saint-Louis, Assistance Publique Hôpitaux de Paris.

\section{$\odot$ Introduction \& Objective}

The fungal flora is acquired early in life. Identification of fungal species for diagnostic or research purposes is a real challenge given their known diversity. For example, ascomycete yeasts are part of the commensal flora. The current barcoding method recommends the use of ITS1/ITS2 primers (ITS1 region) for identification of clinical isolates (1). In metataxonomic study using new generation sequencing, ITS1/ITS2 primers are the most frequently used to evaluate the diversity of fungi in floras and determined the so -called "mycobiome". But other primers such as fITS7/ITS4 have already been evaluated for ecological studies (2). Given the diverging results obtained in mycobiome studies using these ITS1/ITS2 primers, it is unclear which couples of primers are the best to uncover accurately and reproducibly the fungal diversity in clinical samples.

We aim at analyse the parameters influencing ITS amplification in order to select the best couples of primers able to describe fungal diversity accurately .

\section{Results}

\section{SET-UP EXPERIMENTS}

fITS7/ITS4

The 2 DNA extraction procedures tested do not significantly impact results ( $\mathrm{n}=3$ strains)

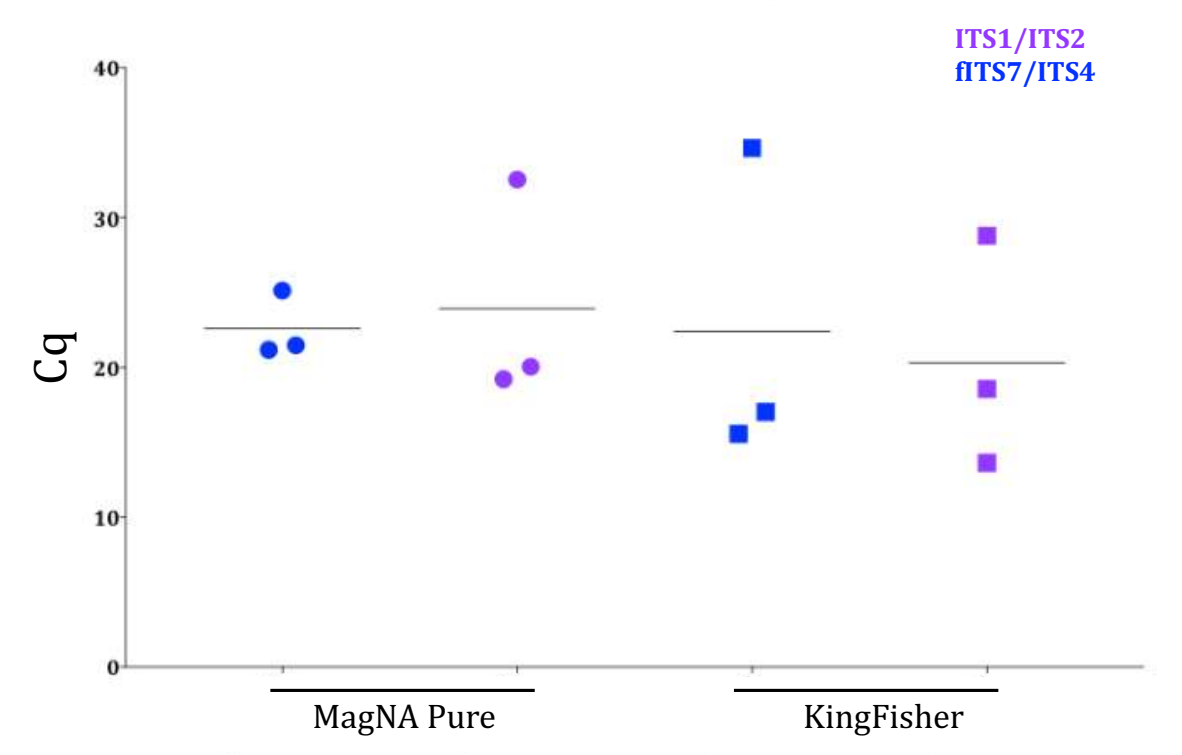

\section{Material \& Methods}

We took advantage of the collection of clinical isolates handled at the French National Reference Center for Invasive Mycoses and Antifungals (NRCMA) and strains from international collection (Westerdijk Fungal Biodiversity Institute, Utrecht, the Netherlands), to select 93 fungal species representing the fungal diversity uncovered in clinical samples. Extraction procedures and DNA quantification methods were first evaluated. All DNA were standardized at $1 \mathrm{ng} / \mu \mathrm{l}$ and screened using primers ITS1/ITS2 and fITS7/ITS4 using the LightCycler® 480 instrument (Roche). We first tested, for 12 strains, the intra species reproducibility by analysing 4 isolates per species. For all qPCR experiments, we used SYBR Green technology with quantification cycles (Cq) and fusion temperature (Tm) as the endpoints.

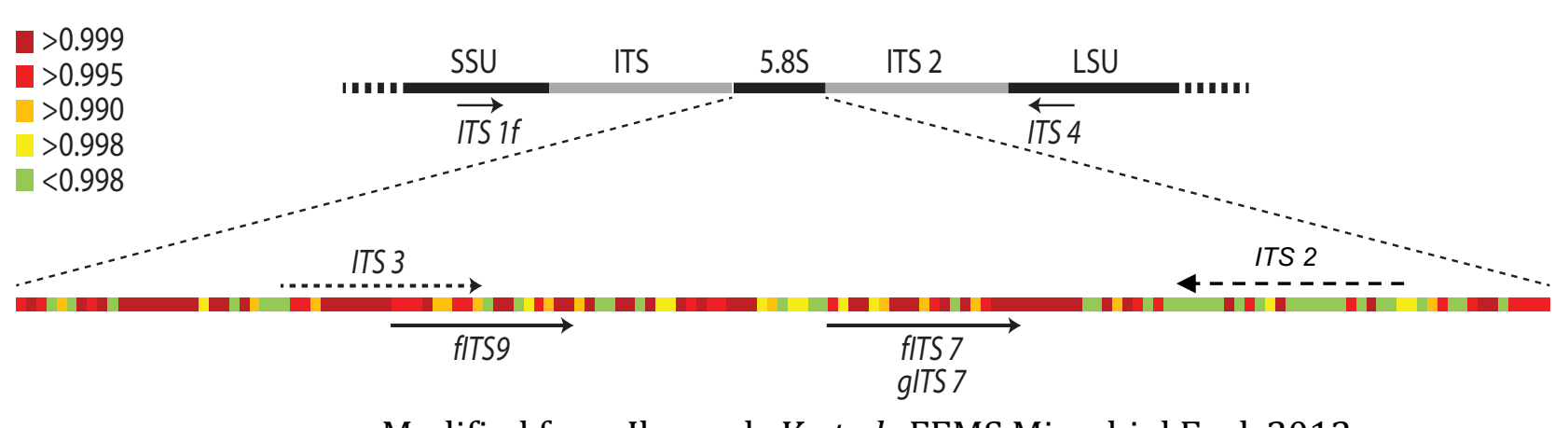

Modified from Ihrmark, K et al., FEMS Microbiol Ecol, 2012
ITS amplification following DNA quantification using Qubit $₫$ fluorimeter is significantly better than using NanoDrop ${ }^{\circledR}$ spectrophotometer $(n=24, p<0.005)$
Storage may influence DNA stability $(n=12)$
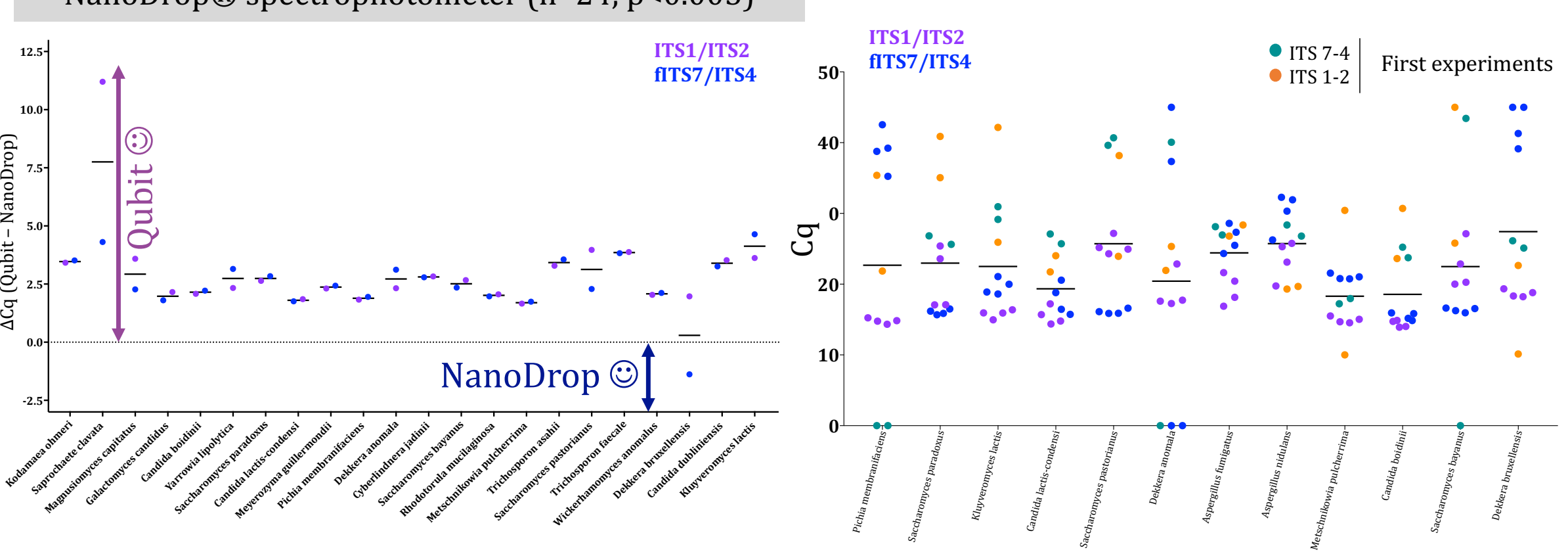

2. ITS1/ITS2 vs. fITS7/ITS4 RESULTS: BASED ON ddCt ANALYSIS ON 93 STRAINS (93 SPECIES, $\mathrm{p}>0.05$ )

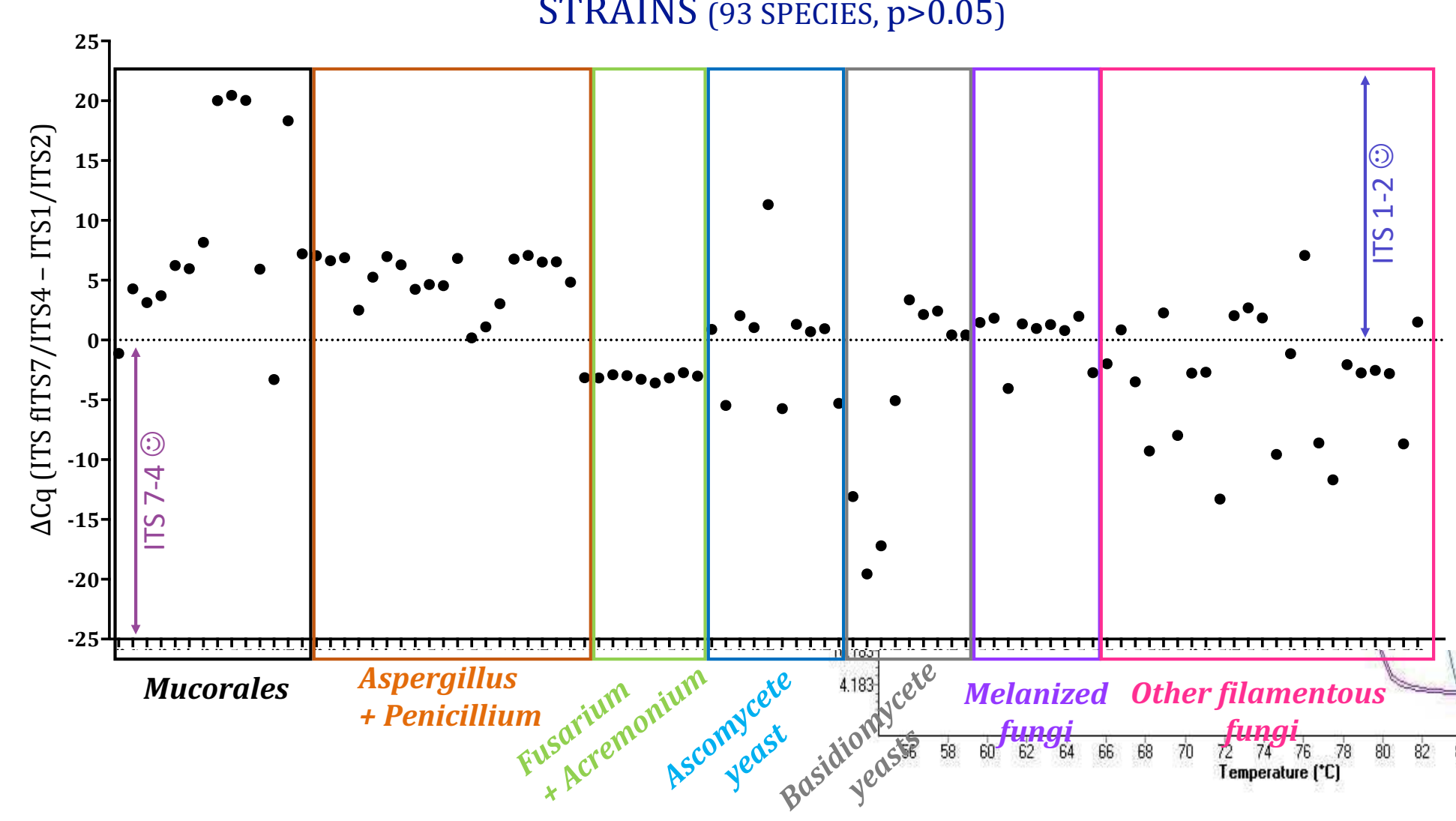

4. NO CONFIRMATION OF THE BETTER AMPLIFICATION ON FOOD YEASTS $(n=24, p>0.05)$$$
\text { ज্ }
$$

5. PREFERENTIAL AMPLIFICATION OF THE STRAIN WITH THE HIGHER Tm VALUE IN CASE OF MIXED

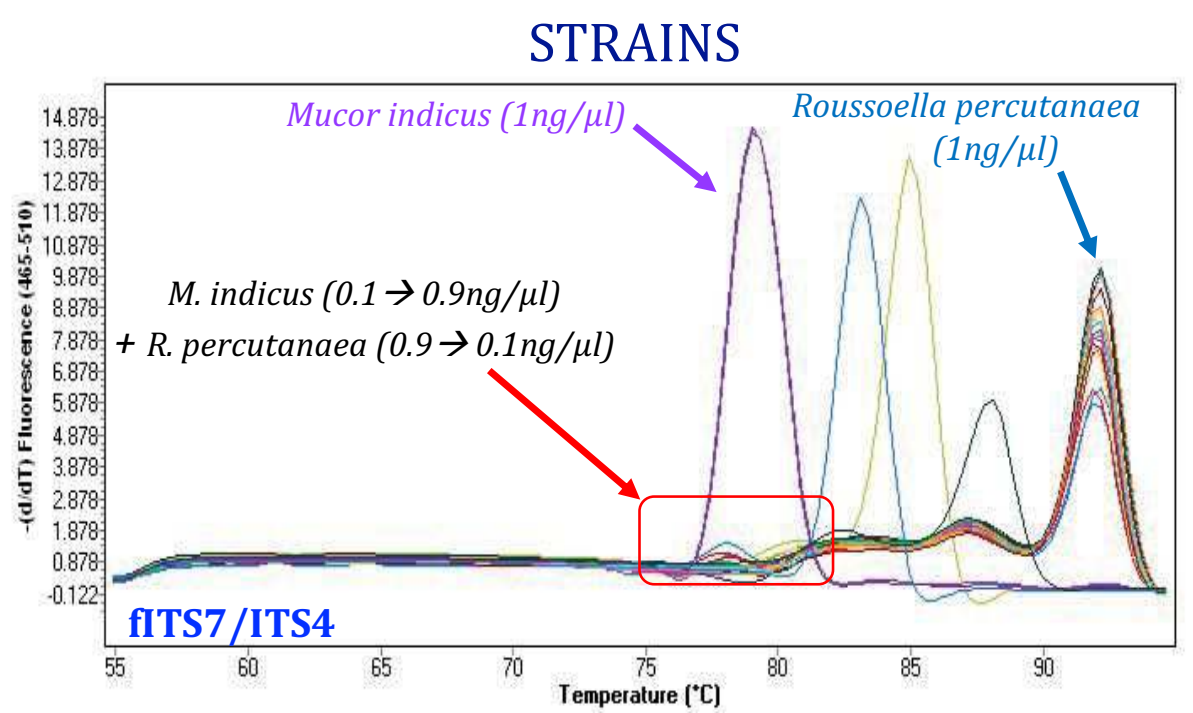

3. BETTER AMPLIFICATION WITH ITS1/ITS2 OR fITS7/ITS4 DEPENDING ON THE FUNGAL GROUPE

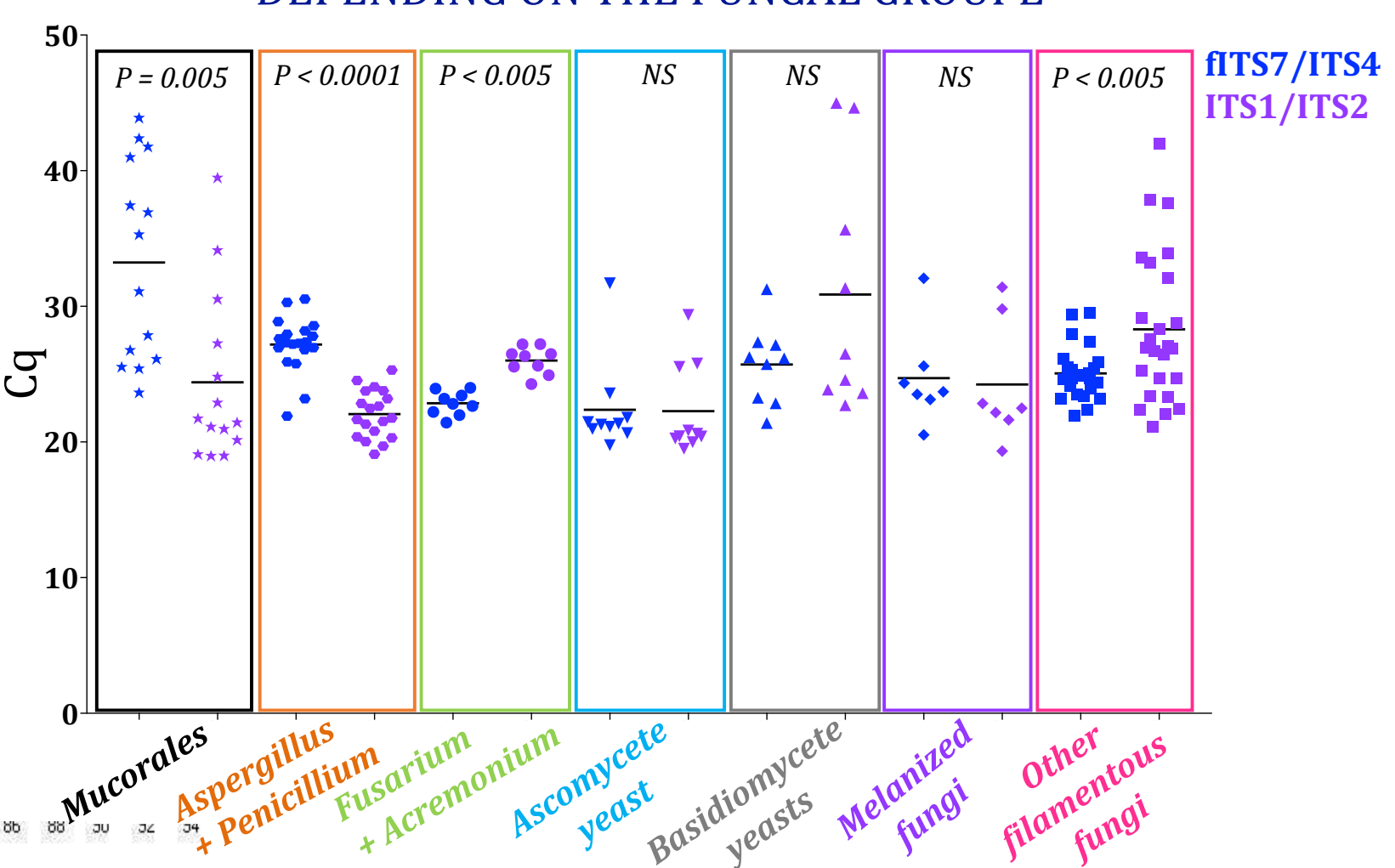

\section{Discussion \& Conclusion}

The choice between ITS1/ITS2 or f7ITS/ITS4 primers to assess fungal diversity is not obvious based on our results showing a preferential amplification for specific group of fungi with each ITS couple of primers. This emphasizes the difficulties to obtain reliable qualitative and quantitative results from metataxonomic studies using ITS amplification and sequencing 